\title{
Immunoglobulin and other proteins in the cerebrospinal fluid of patients with Alzheimer's
} disease

\author{
HM CHAPEL,${ }^{*}$ MM ESIRI, $\dagger$ GK WILCOCK $\dagger$ \\ From the *Immunopathology Department, John Radcliffe Hospital, Oxford, and $†$ Neuropathology and \\ Geriatrics Departments, Radcliffe Infirmary, Oxford
}

SUMMARY Immunoglobulin has been measured and studied electrophoretically in cerebrospinal fluid (CSF) from 14 patients with Alzheimer's disease and 25 undemented controls. Presence or absence of the diagnosis of Alzheimer's disease was confirmed histologically, as these were postmortem specimens. There was no increased incidence of oligoclonal IgG bands in either group, and no significant differences in the levels of IgG and albumin. Non-immunoglobulin bands were found in the gamma region in some samples from Alzheimer's disease patients and controls; such bands are not found in the CSF from younger patients. There was a significantly increased incidence of double transferrin and double tau protein bands in the Alzheimer's group, suggesting that further studies of genetic markers might be worthwhile.

Electrophoretic examination of immunoglobulin in the cerebrospinal fluid (CSF) of patients with progressive dementia has been performed in three studies. Such studies are of interest for the light they may throw on the aetiology of Alzheimer's disease, the commonest cause of senile and presenile dementia. Williams et al' claimed to find oligoclonal IgG in the CSF of five of eight samples examined from patients with presenile dementia. They suggested that this disease may be due to an infectious agent, since other diseases known to be due to infectious agents - such as syphilis and subacute sclerosing panencephalitis - are associated with the presence of oligoclonal IgG bands in the CSF. Other studies have failed to show oligoclonal bands in CSF from demented patients. ${ }^{23}$

We report here an electrophoretic study of CSF obtained from the cerebral ventricles of a group of patients at necropsy. The patients were prospectively assessed for the presence or absence of dementia during life, and histological examination of the brain at necropsy confirmed or refuted the clinical diagnosis of Alzheimer's disease. There was no increased incidence of oligoclonal IgG bands in the CSF from patients with Alzheimer's disease compared with controls. Non-immunoglobulin proteins, however, were different in the CSF samples from the two types of patients.

Accepted for publication 1 March 1984

\section{Material and methods}

Ventricular CSF was obtained at necropsy from 42 patients: 17 of these patients were known from the performance of a mental test score to have been demented during life and 25 were undemented controls. Of the 17 demented patients, 14 had the diagnosis of Alzheimer's disease confirmed by pathological examination of the brain. These brains contained numerous argyrophilic plaques and neurofibrillary tangles in frontal and temporal lobe neocortex and hippocampus. One brain also showed evidence of mild cerebrovascular disease, and one contained a few small inactive multiple sclerosis plaques. Three of the brains from demented patients showed evidence of cerebrovascular disease only, and these patients were then reclassified to the control group. Of the 25 undemented patients, 14 had no relevant central nervous system disease, eight had cerebrovascular disease, and three had evidence of other central nervous system disease (mild hydrocephalus, old contusions, or Parkinson's disease).

Some of these patients have been the subjects of earlier studies. ${ }^{4-6}$ The interval between death and postmortem examination (during which the bodies were refrigerated) varied between $8 \mathrm{~h}$ and four days and did not differ between the groups studied. Mean ages of patients in the two groups were comparable.

CSF specimens were kept frozen until the time of 
assay. IgG and albumin were measured nephelometrically, and polyacrylamide gel electrophoresis was performed according to the method of Thompson $e t$ al. ${ }^{7}$ Gels were stained with both Commassie blue and naphthalene black. Eight specimens in which haemoglobin-haptoglobin complexes, indicative of blood contamination, were detected were excluded from the electrophoretic part of the study (two patients with Alzheimer's disease and six controls).

All the CSF analyses were done without knowledge of the clinical and pathological diagnoses.

Serum samples, obtained at postmortem examination, were available for seven Alzheimer's disease patients and 23 controls.

\section{Results}

Concentrations of IgG and albumin in the CSF of patients with Alzheimer's disease and of controls were unremarkable. The IgG:albumin ratios in CSF from patients with Alzheimer's disease ranged from 9 to $30 \%$ (mean $22 \% ; n=14$ ). The range in control patients was wider $(5-54 \%)$, but the mean was simi$\operatorname{lar}(25 \% ; n=28)$. One in four of the Alzheimer patients had a CSF IgG:albumin ratio greater than $25 \%$; this proportion was similar in the control group (see Table 1).

Serum IgG and albumin concentrations were widely variable in patients with Alzheimer's disease and in controls, but there was no significant difference in these values between the two groups. The wide variation in the serum concentrations made calculation of $\mathrm{IgG}$ indices unreliable. ${ }^{8}$

Electrophoretic analysis showed no excess of oligoclonal bands in Alzheimer's disease CSF compared with controls (Table 2). Oligoclonal IgG bands were found in the CSF of two patients with Alzheimer's disease (17\%) compared with six of the controls $(27 \%)$. The presence of oligoclonal bands was not correlated with a raised IgG:albumin ratio in CSF (Table 1). There was detectable $\operatorname{IgA}$ in the $\gamma$ 1 position in three patients with Alzheimer's disease
Table 2 Comparison of ages and CSF electrophoretic findings between patients and controls*

\begin{tabular}{lcc}
\hline & $\begin{array}{l}\text { Patients with Alzheimer's } \\
\text { disease } \\
(n=12)\end{array}$ & $\begin{array}{l}\text { Controls } \\
(n=22)\end{array}$ \\
\hline Age range (yr) & $51-93$ & $68-99$ \\
mean (yr) & 88 & 81 \\
Oligoclonal IgG & $2(17 \%)$ & $6(27 \%)$ \\
Diffuse IgA & $3(25 \%)$ & $627 \%)$ \\
Acute inflammation & $11(92 \%)$ & $17(77 \%)$ \\
$\gamma 3$ & $8(66 \%)$ & $16(72 \%)$ \\
$\gamma 5$ & $4(33 \%)$ & $4(8 \%)$ \\
Double transferrin & $5(42 \%)$ & 1 \\
Double $\alpha_{1}$-antitrypsin & $3(25 \%)$ & $1(4 \%)$ \\
\hline
\end{tabular}

*CSF contaminated with blood was excluded. (See Material and methods).

and in six of the control group, but the difference was not significant.

Electrophoretic examination of other proteins in the CSF showed acute inflammatory proteins in samples from 11 of 12 patients with Alzheimer's disease $(92 \%)$ compared with 17 of 22 control samples $(77 \%)$, a difference that is not significant. $\overparen{D}$ Single bands in the $\gamma 3$ and $\gamma 5$ positions were found $\vec{\varphi}$ in some samples from patients with Alzheimer's dis- $\stackrel{\infty}{+}$ ease and controls. These did not appear to be $\mathrm{IgG}$ because they stained equally with Commassie blue and naphthalene black. A higher incidence of double transferrin and double tau protein bands was found in CSF samples from the Alzheimer's disease group compared with in those of the control group (Table 2). Of the samples from patients with Alzheimer's disease, $42 \%$ showed a double transferrin band (controls $4 \% ; \mathrm{p}<0.02$ ) and $25 \%$ a double $\alpha_{1}$-antitrypsin band (controls $4 \%$; NS). The presence of these bands did not correlate with delay in performing the postmortem examination or with drug treatment of the patients.

\section{Discussion}

In this study oligoclonal bands in CSF were found in a proportion of patients with Alzheimer's disease

Table 1 Comparison of nephelometric and electrophoretic findings between patients and controls. *

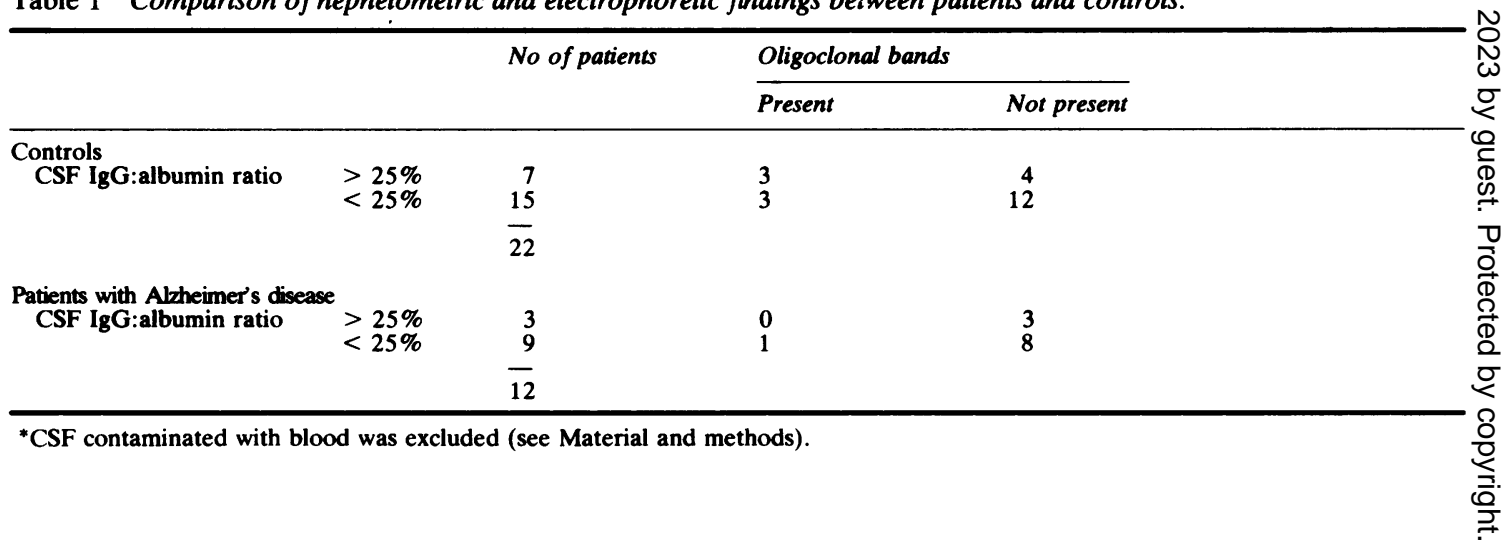


and control patients. The importance of the presence of oligoclonal bands in $17 \%$ of the patients with Alzheimer's disease and $27 \%$ of control patients is uncertain. The latter group included some patients with old or recent strokes and cerebral infarcts at postmortem examination of the brain, and it is of interest that oligoclonal bands have been found in CSF of stroke patients before." Whatever the cause of the oligoclonal bands in our patients' CSF, this study does not support the view that Alzheimer's disease is caused by an infectious agent capable of stimulating an immune response in the central nervous system.

We found an unexpectedly high incidence of unusual CSF bands representing nonimmunoglobulin protein in this study. Our view that these bands are not immunoglobulin rests on the finding that they were stained equally well with both Commassie blue and naphthalene black. Some of the earlier studies of CSF in dementia have not used both stains and it is possible that the bands reported by Williams et al were haptoglobin rather than immunoglobulin.

A band in the $\gamma 3$ position was seen in CSF from $66 \%$ of patients with Alzheimer's disease and $72 \%$ of control patients, and a band in the $\gamma 5$ position was seen in $25 \%$ of patients with Alzheimer's disease and $78 \%$ of controls. Bands in these positions have not been seen in CSF samples from younger patients in our laboratory. Whether they represent serum derived or central nervous system derived proteins remains to be determined. There was no obvious correlation with delay before necropsy but further work will also be required to discover whether these bands may be related to autolysis. Acute inflammatory proteins were often seen in samples of CSF from patients with Alzheimer's disease $(92 \%)$ and controls $(77 \%)$, indicating increased vascular permeability of the blood-brain barrier in both groups.

Double transferrin and double tau proteins were found in the samples from patients with Alzheimer's disease more frequently than those of controls (Table 2). This finding suggests that it may be of some interest to search for other genetic markers in CSF of patients with Alzheimer's disease. HLA typing has so far proved to be disappointing as no specific marker associated with Alzheimer's disease has been shown, but studies of complement $\mathrm{C} 4$ allotypes have suggested an association between a C4 B2 variant and Alzheimer's disease..$^{10-12}$ Other markers may well exist and are not necessarily confined to chromosome 6 .
The advantage of studying postmortem CSF samples has been that it has been possible to study a group of patients with histologically confirmed Alzheimer's disease. Use of postmortem samples of CSF may, however, introduce distortions in patterns of proteins that are due to postmortem autolysis. Nevertheless, the significant differences in the incidences of some protein bands in this series between patients with Alzheimer's disease and controls is encouraging and suggests that further studies of CSF and serum from such patients would be worthwhile.

This work was supported by a grant from the Locally Organised Research Scheme, Oxford RHA.

We are grateful to Mrs J Wilkinson and Mr D Smith for their excellent technical assistance, and to Dr EJ Thompson of the Institute of Neurology, London.

\section{References}

' Williams A, Papadopolos N, Chase TN. Demonstration of the CSF gammaglobulin banding in presenile dementia. Neurology 1980;30:882-4.

${ }^{2}$ Jonker C, Eikelenboom P, Tavenier P. Immunological indices in the cerebrospinal fluid of patients with presenile dementia of the Alzheimer type. Br J Psychiatry

${ }^{3}$ Chu AB, Sever JL, Madden DL, et al. Oligoclonal IgG bands in cerebrospinal fluid in various neurological diseases. Ann Neurol 1983;13:434-9.

${ }^{4}$ Wilcock GK, Esiri MM. Plaques, tangles and dementia; a quantitative study. J Neurol Sci 1982;56:343-56.

${ }^{5}$ Wilcock GK, Esiri MM, Bowen DM, Smith CCT. Alzheimer's disease. Correlation of cortical choline acetyltransferase activity with a severity of dementia histological abnormalities. $J$ Neurol Sci 1982;57:407-14.

- Appleyard ME, Smith AD, Wilcock GK, Esiri MM. Decreased acetyl choline activity in Alzheimer's disease. Lancet 1983;ii: 2452.

'Thompson EJ, Kaufman P, Shortman RC, Rudge P, McDonald WI. Oligoclonal immunoglobulins and plasma cells in spinal fluid of patients with MS. Br Med J 1979;i:16-7.

${ }^{8}$ Thompson EJ, Riches PG, Kohn J. Antibody synthesis within the central nervous sytem: comparisons of CSF IgG indices and electrophoresis. J Clin Pathol 1983;36:312-5.

${ }^{9}$ Rostrom E, Link H. Oligoclonal immunoglobulin in cerebrospinal fluid in acute cerebrovascular disease. Neurology 1981;31:590-6.

${ }^{10}$ Henschke PJ, Bell DA, Cape RDT. Alzheimer's disease and HLA. Tissue Antigens 1978;12:132-5.

" Sulkava R, Koskimies SM, Wikstrom J, Palo J. HLA antigens in Alzheimer's disease. Tissue Antigens 1980;16:191-4.

${ }^{12}$ Nerl CW, Mayeux R, O'Neil GJ. Complement C4 allotypes in Alzheimer's disease. Lancet 1982;ii: 1343.

Requests for reprints to: Dr HM Chapel, Department of Immunology, John Radcliffe Hospital, Oxford. 